\title{
Factores de riesgo y escalada cannabinoide
}

\author{
VAzQuez, F; Becoña, E. \\ Universidad de Santiago de Compostela
}

Enviar correspondencia a: F.L. Vázquez. Universidad de Santiago de Compostela. Facultad de Psicología.

Departamento de Psicología Clínica y Psicobiología. Campus Universitario Sur. 15702 Santiago de Compotela.

\begin{abstract}
Resumen
El objetivo de esta revisión es analizar los predictores de uso y abandono del consumo de cannabis, la hipótesis de la escalada, los conceptos de causalidad y probabilidad y, especialmente, los factores de riesgo relacionados con la escalada cannabinoide. Estudios realizados en distintos países han confirmado que existe una relación significativa entre el consumo de sustancias legales, alcohol y tabaco, en consumidores de cannabis respecto a los que no consumen. También hay evidencia del papel del cannabis como una sustancia que facilita la escalada para el abuso de drogas como la heroína o la cocaína. En el proceso de escalada intervienen también otros factores tales como la personalidad, el estilo de vida y distintos factores ambientales. La iniciación al consumo de cannabis, además de estar relacionado con el consumo previo de alcohol y cigarrillos, está relacionado principalmente con la edad del joven, ser varón, baja satisfacción escolar, bajo rendimiento académico, sentirse no querido por la familia, problemas de salud mental, no ser popular y formar parte de un grupo de compañeros que consumen drogas.
\end{abstract}

Palabras clave: cannabis, factor de riesgo, escalada.

\section{INTRODUCCIÓN}

E I la última encuesta nacional que se ha hecho sobre el consumo de drogas en una muestra representativa de la población escolar de 14 a 18 años (Plan Nacional sobre Drogas, 1997), se encontró que las edades de inicio de las distintas sustancias son de 13.3 años para el tabaco,

\section{Abstract}

The purpose of this review is to analyse cannabis consumption and cessation predictors, the steppingstone hypothesis, the concepts of causality and probability, and, particularly, the risk factors related to cannabis escalation. The studies carried out in various countries confirm the existence of a significant relationship between the consumption of legal substances (alcohol and tobacco) and cannabis consumption. There is also evidence of the role of cannabis in facilitating the escalation towards the abuse of other drugs like heroine of cocaine. Other factors intervene in the process of escalation such as personality, lifestyle, and other environmental factors. The initiating of cannabis consumption, besides being related with a prior consumption of alcohol and cigarettes, is mainly related to the young person's age, being male, low school satisfaction, low academic achievement, feeling unloved by family, mental health problems, lack of popularity, and belonging to a peer group that consumes drugs.

Key words: cannabis, risk factors, stepping-stone hypothesis.

13.7 para el alcohol, 14.9 para el éxtasis, 15.0 para el cannabis, 15.4 para los alucinógenos, 15.5 para las anfetaminas y 15.7 para la cocaína. Aunque de menor importancia cuantitativa, el inicio al consumo de las sustancias volátiles es más temprano, a los 13.1 años. Además, hay un incremento del consumo de tabaco, alcohol y otras sustancias en función de la edad, especialmente a partir de los 13 años. 
En los jóvenes europeos que "salen de marcha", la edad media de inicio para las distintas drogas es de 14.6 años para el alcohol, 14.8 para el tabaco, 16.0 para el cannabis, 17.7 para las anfetaminas y el LSD, 18.4 para el éxtasis y 19.3 para la cocaína (Calafat et al., 1999).

Los datos de estos dos estudios retrospectivos, así como los encontrados en otros del mismo tipo (ej., O'Malley et al., 1999), indican que la experiencia inicial con las sustancias ocurre de modo típico a la edad de 13 años, aunque no se debería olvidar que una parte comienzan antes de dicha edad. En la adolescencia tardía, la prevalencia de vida de algunas sustancias es importante, mientras que el consumo excesivo es considerablemente más bajo. De ello se infiere que un subgrupo de adolescentes se implica en un consumo intenso de sustancias, mientras que otros sólo se implican en una mínima experimentación.

El término "escalada" se utilizó como un símil del montañismo, que se aplicó al proceso por el cual quien se iniciaba en el consumo de drogas (en referencia, generalmente, a los derivados del cannabis) progresaba secuencialmente hasta la heroína. El cada vez mayor conocimiento de la realidad de las drogas permitió ir matizando esta generalización. Hoy se sabe que no existe un encadenamiento bioquímico por el que de unas sustancias se deba ir pasando necesariamente a otras de mayor capacidad adictiva. Parece más razonable hablar de una relación de probabilidad entre los distintos consumos (Becoña, 1999). Así, aquellos adolescentes que tengan un consumo más intenso y precoz de alcohol y tabaco, tienen más probabilidades de iniciarse en el consumo de cannabis. Así mismo, aquellas personas que, siguiendo este proceso, se inicien precozmente en el consumo de cannabis, es más probable que se impliquen en el uso de otras drogas ilegales.

Actualmente los estudios longitudinales sugieren que un subgrupo de adolescentes es vulnerable al consumo de sustancias de forma escalada, siendo el cannabis la "puerta de entrada" a otras drogas ilegales (ej., heroína, cocaína). El consumo de cannabis forma parte de un continuo que incluye el consumo experimental, ocasional, diario y crónico (Gold y Tullis, 1999). Dicha sustancia es generalmente la primera droga ilegal consumida por la gente joven (Millman y Beeder, 1997).

El objetivo de esta revisión es analizar los predictores de uso y de abandono del consumo de cannabis, la hipótesis de la escalada, los conceptos de causalidad y probabilidad y distintos factores de riesgo relacionados con la escalada y el consumo de cannabis.

\section{PREDICTORES DE USO, ABUSO Y ABAN- DONO DEL CANNABIS}

Existen distintos patrones de uso y abuso asociados con el cannabis. El nivel de abuso parece depender del país al que pertenece el individuo, aunque hay algunas variables que se deberían considerar (Gold y Tullis, 1999): 1) demográficas (edad, sexo, localización geográfica); 2) factores sociales (factores multiculturales, estatus socioeconómico, influencia de los padres, nivel cultural); y, 3) factores individuales (estado psicológico, emocional y fisiológico).

Se han estudiado numerosas variables para explicar el consumo del cannabis en la adolescencia. Por ejemplo, los estudiantes de bachillerato que obtienen buenos resultados académicos, que están implicados en actividades atléticas o de la escuela, que tienen importantes creencias religiosas, trabajan pocas horas fuera de la escuela y salen poco por las tardes durante la semana tienen menos probabilidades de llegar a consumir cannabis (Gold y Tullis, 1999). El consumo de cannabis está relacionado con frecuencia con el contexto social y la aceptación de esta sustancia por parte de los compañeros. Las interacciones con drogas y usuarios de drogas también son un factor de riesgo importante.

Datos recientes sugieren que el uso del cannabis y alcohol en los adolescentes se asocia con conductas de control de peso inadecuadas (Neumark-Sztainer et al., 1998). Aunque los factores genéticos parecen tener un mínimo efecto en la probabilidad de usar 
cannabis, afectan a la probabilidad de abuso y dependencia (Gold y Tullis, 1999). Otra posible razón para el consumo podría estar relacionada con la función del tetrahidrocannabinol (THC) en modificar la función cerebral y la de distintas hormonas (Díaz, 1997; Martin, 1999). El contenido en THC de la marihuana continúa cambiando y también puede jugar un papel en el incremento del consumo de dicha sustancia en los adolescentes. A principios de los años 70, un cigarrillo de marihuana tenía aproximadamente una concentración del 1 al 3\% de THC, o lo que es lo mismo, de 5 a $30 \mathrm{mg}$. En la actualidad, y debido a las mejoras en el tipo de planta cultivada, la riqueza en cannabinoides puede llegar a alcanzar hasta los $150 \mathrm{mg}$ por cigarrillo, incluso el doble si lo que se fuma es la resina (Cabrera y Torrecilla, 1998). En estudios controlados se ha demostrado que a mayor contenido en THC más usuarios lo prefieren y lo autoadministran (Kelly et al., 1994). H a y otros factores que también pueden tener relación con el consumo de cannabis tales como una disminución en el coste, escasa desaprobación del consumo por parte de los compañeros y baja percepción de riesgo (Bachman et al., 1997). Las experiencias positivas con una sustancia psicoactiva, o el reconocimiento de que la experiencia no es tan peligrosa como han indicado los medios de comunicación o los padres, puede alentar la experimentación con otro tipo de drogas. Puesto que el consumo de cannabis es ilegal e implica un comportamiento desviado, cabe que se reduzcan las barreras para utilizar otras sustancias ilegales, que están más desaprovadas por la autoridad convencional (Millman y Beeder, 1997).

En cuanto a los factores relacionados con el abandono, la edad es uno de los predictores más importantes. La madurez se asocia a intereses, necesidades y actitudes diferentes, incluyendo la disminución de la implicación en conductas de riesgo y en un incremento en la conformidad. Las características de la adolescencia relacionadas con el uso de sustancias tales como realizar conductas de riesgo, actividades delictivas, baja religiosi- dad, depresión y baja autoestima son menos probables que aparezcan en la adultez. Parece ser que hay una relativa incompatibilidad entre el uso de drogas ilegales y la participación en los roles convencionales de la adultez (Chen y Kandel, 1998).

En el estudio reciente de Chen y Kandel (1998) encontraron que los dos predictores más importantes para dejar de fumar marihuana fueron la frecuencia de uso y la edad. Los usuarios de poca frecuencia y aquellos que pasaban de los 20 años era más probable que dejaran de consumir marihuana. Los usuarios frecuentes eran más persistentes en su uso y muchos llegaban a ser dependientes. El consumir por razones sociales aceleraba el abandono, mientras que el consumir para intensificar los sentimientos positivos y para reducir los negativos se asoció con la persistencia del consumo. Muchos de esos fumadores perdieron su costumbre de fumar como resultado de la madurez, formar una familia, la educación y el empleo.

\section{LA HIPÓTESIS DE LA ESCALADA}

Uno de los modelos más conocidos en el campo de las drogodependencias es el de Denise Kandel. Este modelo se basa en que en el consumo de drogas hay unos pasos secuenciales, siendo el comienzo por las drogas legales, las cuales facilitarían el posterior consumo de marihuana, que a su vez, sería la "puerta de entrada" para el consumo de otras drogas ilegales. Para Kandel (1975) hay al menos cuatro estadios de desarrollo en el uso de las drogas: 1) consumo de cerveza o vino; 2) cigarrillos o licores; 3) marihuana; y, 4) otras drogas ilegales (ej., heroína, cocaína). Por tanto, el consumo de drogas legales es el elemento intermedio entre el no consumo de ninguna sustancia y el consumo de marihuana, y éste, conduce o es la "puerta de entrada", para un porcentaje significativo de sus consumidores de heroína o cocaína.

La mayoría de la evidencia de este modelo deriva del trabajo que ha llevado a cabo por el equipo de investigación de la doctora Kandel 
en las dos últimas décadas y, en particular, del seguimiento longitudinal que se ha hecho a una cohorte de adolescentes durante 19 años (hasta la cuarta década de la vida), el "New York State Cohort". En 1971-72 se reclutó una muestra representativa de 1.222 jóvenes estudiantes de 101 y 111 grado (equivalente al 41 curso de la ESO y al 11 curso de bachillerato impartido en España actualmente) en las escuelas públicas de Nueva York. Los participantes fueron muestreados dos veces en el Instituto y se les volvió a entrevistar en 1980, en 1984 y en 1990. Los sujetos fueron reclutados cuando tenían 15-16 años y se les siguió hasta los 34-35 años. En cada momento de la evaluación se llevaron a cabo entrevistas personales estructuradas de aproximadamente dos horas de duración. En ellas evaluaron siete aspectos principales: variables demográficas, historia familiar de trastornos psiquiátricos y problemas con el alcohol, relaciones y actitudes de los padres, relación con los compañeros, participación en actividades delictivas, síntomas psicológicos y variables relacionadas con las drogas. En cada informe se recogió información sobre la historia de consumo de 12 drogas o clases de drogas: dos legales (cigarrillos y alcohol), cuatro ilegales (marihuana, psicodélicas, cocaína y heroína) y seis clases de drogas psicotrópicas de uso y no uso médico: metadona, tranquilizantes menores y mayores, sedantes, estimulantes, antidepresivos y otros opiáceos diferentes de la heroína.

Los datos de este estudio permitieron aislar dos tipos de predictores de la progresión en la secuencia de implicación con las drogas: 1) características conductuales de la historia individual con la droga; $y, 2$ ) predictores psicológico-sociales. En el primer grupo se encontró que las variables más predictivas fueron la edad de comienzo de consumo de las drogas legales y el grado de implicación con el consumo de la misma. El comienzo temprano del consumo de alguna droga estaba asociado con una mayor implicación en el uso de todas las otras drogas (Kandel y Yamaguchi, 1985; Yu y Williford, 1992). Cuanto más temprano se producía la experimentación con las drogas legales, mayor probabilidad había de que el joven también experimentase con drogas ilegales y progresase a una implicación mayor con estas sustancias. En concreto, las proporciones de varones entre 34-35 años que habían experimentado con la marihuana oscilaron entre el $85 \%$ de los que informaron que habían probado el alcohol o los cigarrillos por primera vez a los 14 años o antes, al $46 \%$ de los que habían probado el alcohol a los 18 años de edad. De modo similar, cuanto más temprano era el consumo de marihuana, había una mayor implicación y una mayor probabilidad de progresar a otras drogas ilegales. Casi todos los hombres jóvenes $(96 \%)$ que se iniciaron en el consumo de la marihuana a los 14 años o a edades más tempranas habían usado otra droga ilegal diferente a los 29 años. La proporción declinó al $66 \%$ entre los que habían probado por primera vez la marihuana a los 18 años de edad y al $33 \%$ entre los que la probaron por primera vez a los 20 años; solamente el $6 \%$ habían consumido alguna droga ilegal distinta de la marihuana sin haber usado ésta nunca. Las proporciones entre las mujeres fueron $84 \%$, $46 \%, 43 \%$ y $9 \%$, respectivamente.

También se encontró que la progresión a una droga superior en la jerarquía estaba relacionada con la intensidad del uso de una droga inferior en la jerarquía (Kandel y Yamuchi, 1985). De modo más específico, el $82 \%$ de los hombres y el $60 \%$ de las mujeres que habían consumido marihuana 1.000 o más veces a las edades de 24-25 años comenzaron a usar otras drogas ilegales en los siguientes cuatro años comparado con el $18 \%$ y el $17 \%$, respectivamente, de los que la habían consumido de 10 a 99 veces.

En cuanto a los predictores psicológicosociales, se examinaron cuatro grupos de ellos (Kandel y Davies, 1992): 1) las influencias de los padres; 2) las influencias de los compañeros; 3) la implicación de los adolescentes en varias conductas; $y, 4)$ las creencias y los valores. La influencia de los compañeros parece ser más importante en ciertos puntos en el proceso de implicación con la cadena adictiva; en ciertas edades, la conducta de los compa- 
ñeros es especialmente importante en predecir el consumo de la marihuana y menos importante para predecir el consumo de alcohol u otras drogas ilegales diferentes de la marihuana. La influencia de los padres es más importante para la progresión a otras drogas ilegales diferentes de la marihuana. La gente joven en riesgo de iniciar el consumo de marihuana realizan más conductas desviadas que sus compañeros. Por último, el uso de la marihuana es más probable cuando la persona acepta una serie de creencias y valores favorables al uso de la misma.

Es más, en un estudio (Golub y Johnson, 1994) se encontró que el alcohol está perdiendo importancia como condición previa para la progresión a la marihuana, pero el papel de la marihuana como "puerta de entrada" a otro tipo de drogas ilegales parece que se está incrementando, y el consumo de marihuana casi siempre precede al uso de sustancias relacionadas con actitudes menos normativas (ej., heroína).

Se debería hacer énfasis en que, aunque se ha identificado una secuencia clara en el desarrollo de la implicación con las drogas, el consumo de una droga es un estadio particular en la secuencia, que no conduce siempre al uso de otras drogas que se encuentran en una posición superior en la jerarquía de esa secuencia (Kandel, Yamaguchi y Chen, 1992). Muchos jóvenes se quedan en un determinado estadio y no progresan a otros. La noción de estadios en la conducta de consumir drogas no implica que estos estadios sean obligatorios o universales (Kandel y Yamaguchi, 1999). El uso de una droga en un estadio inferior es necesario pero no suficiente para progresar a un estadio superior en la jerarquía en el que se consuman otro tipo de drogas (O’Donnell y Clayton, 1982).

\section{CAUSALIDADY PROBABILIDAD}

A menudo el fenómeno de la escalada se ha interpretado inadecuadamente. Se ha partido de que todos los adolescentes que terminan siendo adictos a drogas ilegales han seguido secuencialmente los pasos previos de la cadena. Este razonamiento es erróneo, se están infiriendo relaciones de causalidad en las variables de este modelo. Más de una vez se ha cometido el error de interpretar los resultados de un análisis de asociación entre variables, dándole un significado que no tiene. Una cosa es la existencia de una fuerte asociación o correlación entre dos o más variables, y otra muy diferente, es la existencia de una relación causal entre las mismas. En el campo de la prevención de las drogodependencias se ha demostrado la existencia de asociación entre muchas variables, pero pocas que tengan una relación causal. Solamente se puede demostrar causalidad a través de una situación experimental (Hulley y Cummings, 1988; Moore y McCabe, 1997). El tipo de diseños que se pueden utilizar para probar la hipótesis de la escalada sólo sirven para conocer si hay asociación o correlación entre el consumo de drogas legales e ilegales en los jóvenes, pero no para concluir que hay causalidad. Aun así, aunque no se pueda llevar a cabo un experimento, en algunas circunstancias se podría llegar a inferir una relación causal de una asociación entre dos o más variables (Hulley y Cummings, 1988): 1) cuando los resultados son consistentes entre estudios que han utilizado diseños diferentes (ej., longitudinales, transversales). En este caso es poco probable que el azar o algún tipo de sesgos sean los que expliquen la asociación entre las variables; 2) cuando se encuentra una fuerte asociación entre las variables, pues una fuerte asociación da más valores p significativos, haciendo que el azar sea una explicación poco probable para la relación de las variables; 3 ) cuando la odds ratio es mayor que 2.5 es poco probable que variables de confusión puedan explicar la asociación; 4) la relación dosis-respuesta también proporciona evidencia de una posible causalidad; y, 5) se puede hacer una inferencia causal cuando hay una causa biológica verosímil que pueda producir un efecto determinado. 
Por otro lado, cuando hablamos de una relación de asociación o de causalidad tenemos que hablar en términos de probabilidad. Los valores de la probabilidad oscilan entre 1 y 0 (Moore y McCabe, 1997). Un valor de 1 equivale a certeza absoluta, y el 0 indica que no hay posibilidad de que ocurra el suceso. Existen muy pocas cuestiones en prevención de drogas, al igual que en la mayoría de los campos del conocimiento, de las que podamos estar completamente seguros, es decir, asumir una probabilidad del $100 \%$. Por lo tanto, la mayoría de lo que se estudia en prevención o en relación con el tratamiento, tienen valores de probabilidad de que ocurran, en algún punto entre 1 y 0 . Si nosotros, por ejemplo, llevamos a cabo un estudio sobre la conducta de consumo de drogas de un adolescente, se pueden plantear dos opciones: 1) que pueda consumir droga; $y, 2$ ) que no la consuma. La probabilidad teórica de la primera opción es de 0.5. También es cierto que la probabilidad de que se dé la segunda opción es 0.5 (no nos interesa el caso en que haya factores de protección o de riesgo que alteren estas probabilidades). Obsérvese que la suma de las dos probabilidades es igual a 1. La probabilidad de que ocurra la primer opción vamos a denominarla $\mathrm{p}$ y la probabilidad de que no ocurra vamos a llamarle q. La suma de $p+q$ es siempre igual a 1. )Cuál es la probabilidad a la que nos estamos refiriendo? De acuerdo a las concepciones probabilísticas de causalidad, las causas incrementan las probabilidades de sus efectos. Esto es, significa que $\mathrm{p}$ (o la presencia del factor $\mathrm{X}$ ) incrementa la probabilidad de $Y$ (o la presencia del factor Y). Por lo tanto, esto aplicado a la hipótesis de la escalada significa que el uso de una droga previa no implica inevitablemente pasar a ser consumidor de la siguiente. Más específicamente, en lo que se refiere al papel de la marihuana en el proceso de escalada, aquellos que consumen marihuana es probable que hayan utilizado tabaco y alcohol, y aquellos que consuman otras drogas ilegales, es probable que hayan usado previamente marihuana.

\section{FACTORES DE RIESGO RELACIONADOS CON LA ESCALADA EN EL CONSUMO DE SUSTANCIAS: PAPEL DEL CANNABIS}

Estudios realizados en distintos países, entre ellos en España (ej., Recio, 1995) han confirmado que existe una relación significativa entre el consumo de sustancias legales, alcohol y tabaco, en consumidores de cannabis respecto a los que no consumen. También hay evidencia del papel del cannabis como una sustancia que facilita la escalada para el abuso de drogas como la heroína o la cocaína. Si se previene el consumo de tabaco y alcohol se reduce la probabilidad de consumo de cannabis y, viceversa, la prevención del consumo de cannabis previene el consumo de otras drogas ilegales (Becoña, 1999).

En el proceso de escalada intervienen otros factores además del propio consumo de la sustancia. El consumo de una droga específica no sólo está relacionado con el consumo previo de otras drogas, en función de la jerarquía propuesta por Denise Kandel, sino que hay otros factores que intervienen en esa relación tales como la personalidad, el estilo de vida y distintos factores ambientales. La iniciación al consumo de cannabis, además de estar relacionada con el consumo previo de alcohol y cigarrillos, también está relacionada con la edad del joven, ser varón, baja satisfacción escolar, bajo rendimiento académico, sentirse no querido por la familia, no ser popular y formar parte de un grupo de compañeros que consumen drogas (Yamaguchi y Kandel, 1985; Werch y Anzalone, 1995).

Hay varios estudios relevantes que ejemplifican la importancia de estas otras variables en el consumo del cannabis. Kandel y Davies (1992) hicieron un seguimiento de una muestra de estudiantes de secundaria hasta la adultez para comprobar la evolución en el consumo de marihuana. Las variables más influyentes para llevar a cabo esta conducta fueron el inicio temprano al consumo, tener compañeros con conductas desviadas, baja participación religiosa, bajas expectativas educativas y tener padres consumidores de sustancias. 
Recio (1995) encuentra en su estudio que las actitudes de los padres hacia el hachís es el mejor predictor de la iniciación al uso del tabaco de sus hijos, así como una actitud percibida como más tolerante por parte de los padres. Sin embargo, para la iniciación del uso del cannabis, las variables más predictivas son el uso del cannabis por parte de los amigos y el rendimiento académico.

Hammer y Vaglum (1990) Ilevaron a cabo un estudio en Noruega con jóvenes entre 17 y 20 años. Encontraron un mayor consumo de cannabis en los sujetos que vivían en la capital de Oslo, en los varones, en aquellos cuyos padres estaban divorciados o vivían con un solo padre o sin sus padres biológicos, dejar tempranamente la escuela, no tener empleo, hacer menos actividades en el tiempo de ocio, hacer menos ejercicio, tener menos contactos sociales con sus amigos y estar menos integrados en el grupo y tener más problemas de salud (somáticos o psicológicos). De todos estos factores, se encontró que los que tenían una mayor relación con el consumo de cannabis, por orden de significación, fueron el tener problemas de salud mental, tener padres divorciados, abandonar tempranamente la escuela y no tener empleo.

Stenbacka et al. (1993) analizaron en una muestra de varones la relación que existía entre el ofrecimiento de drogas, el consumo de cannabis y la progresión de esta sustancia al abuso de drogas por vía intravenosa. El estudio se llevó a cabo en Suecia, en los años 1969-70, sobre una cohorte de 50.462 jóvenes aptos para realizar el servicio militar, de los que se incluyeron en el estudio 23.482 jóvenes. Se evaluaron variables demográficas, familiares, adaptación escolar, relaciones con los amigos, consumo de alcohol, tabaco, narcóticos y solventes esnifados; igualmente, si utilizaban drogas de modo intravenoso. También se evaluaron variables psicológicas de control emocional, madurez social y funciones psíquicas.

Del total de la muestra, al $42 \%$ les habían ofrecido drogas ilegales y el $10.7 \%$ las probó. La droga más probada fue el cannabis, en el $84 \%$ de los casos. De éstos, el $8 \%$ usaba también drogas por vía intravenosas. Se encontró que el consumo de sustancias como el alcohol, el tabaco y solventes incrementaba la probabilidad de consumo de marihuana y de drogas inyectadas. Se halló una notable relación entre el cannabis y el abuso de drogas por vía intravenosa. Aquellos que habían utilizado el cannabis más de 5 veces presentaron una odds ratio de 15.9 en el análisis univariado, mientras que para los que lo usaron entre 1 y 4 veces fue de 1.0.

Por otra parte, en aquellos que habían utilizado drogas ilegales se detectó una alta proporción de desajuste social, bajo control emocional, abandono del hogar, uso de solventes esnifados y problemas con la policía. Los factores de riesgo para el cannabis, por orden de significación, fueron el abuso de solventes, el haber abandonado el hogar, el fumar más de cinco cigarrillos diarios, problemas con la policía o con las autoridades judiciales, hábito de consumo de alcohol del padre, embriagarse, bajo control emocional y el tener una economía familiar muy mala. Los factores de riesgo para el abuso de sustancias por vía intravenosa fueron semejantes, destacando por el tamaño de la odds ratio, el abandono del hogar y tener problemas con la policía.

El estudio de Stenbacka et al. (1992) con una muestra de 8.168 jóvenes suecos mostró, respecto al consumo de cannabis, que el abuso del mismo en el pasado se asoció con un mayor riesgo de consumo de drogas actual (odds ratio $=3.6$ )

Wills et al. (1997) analizaron el fenómeno de la escalada en una muestra de adolescentes a los que siguieron durante tres años. A lo largo de este período llevaron a cabo tres evaluaciones (en la primera evaluación la edad media de la muestra fue de 12.4 años), en base a las cuales pudieron identificar varios grupos, utilizando el análisis estadístico "cluster": 1) no consumidores estables, que eran los que no habían consumido ninguna sustancia a lo largo del tiempo; 2) experimentadores mínimos, aquellos que habían tenido un consumo mínimo; 3) comienzo tardío, que eran los que experimentaban con las sustancias en los grados $7^{\circ}$ y $8^{\circ}$, pero lo incremen- 
tan en el grado $9^{\circ}\left(1^{\circ}, 2^{\circ}\right.$ y $3^{\circ}$ de la ESO en España, respectivamente); 4) escalador 1 , que eran aquellos que ya tenían un consumo elevado de sustancias cuando estaban cursando el grado 71 y lo seguían incrementando a lo largo del tiempo; y, 5) escalador 2, aquellos que se habían iniciado muy tempranamente al consumo y que lo habían incrementado de modo acusado a lo largo del tiempo. Las sustancias que consumían eran tabaco, alcohol y marihuana. Se encontró que los escaladores tenían un gran número de factores de riesgo y escasos factores de protección. Los más significativos fueron el experimentar un mayor estrés vital, el bajo apoyo parental, el mayor uso de sustancias por parte de los padres, tener actitudes desviadas y habilidades de afrontamiento desadaptativas, bajas habilidades de autocontrol y mayor afiliación con iguales que consumían sustancias, especialmente la marihuana.

Otro importante factor de riesgo del consumo de cannabis se asocia con la vida recreativa actual y la normalización de los consumos de las llamadas drogas "recreativas" (Calafat et al., 1999), no viendo a estas como un problema o que puedan causarles problemas, sino más bien como una ayuda eficaz para aguantar "la marcha". El policonsumo se hace así un elemento más de "la marcha", donde junto al tabaco y el alcohol están el cannabis, las drogas de síntesis y la cocaína. El entretenimiento se asocia con frecuencia al uso de drogas, especialmente cuando hay que cambiar los horarios habituales y hay que trasnochar muchas horas, sobre todo cuando la diversión dura hasta la mañana del día siguiente.

\section{CONCLUSIÓN}

Las personas implicadas en el consumo de drogas siguen una secuencia bien definida (Kandel y Yamaguchi, 1999). El alcohol y/o el tabaco, drogas que son legales para los adultos de nuestra sociedad, son las sustancias que primero se consumen. El consumo del cannabis rara vez tiene lugar sin haber consu- mido previamente alcohol o tabaco o ambas $y$, el consumo de drogas ilegales, que no sea el cannabis, es raro en ausencia de una experimentación previa con el mismo.

Actualmente se considera que hay algunos factores de riesgo que pueden intervenir en el fenómeno de la escalada, tal como hemos analizado anteriormente. Como comentan Hammer y Vaglun (1991), concretamente sobre el cannabis, la investigación debe dirigirse a analizar los factores de personalidad y los procesos sociales que llevan a los jóvenes que viven en un contexto de alto riesgo a ser capaces de no llegar a consumir sustancias como el cannabis u otras. De las palabras de estos autores se infiere claramente que tenemos un conjunto clave de factores de riesgo que se asocian con el uso del cannabis. Algunas de ellos los propusieron dichos autores: "Los factores principales que discriminan entre los adultos jóvenes que han consumido cannabis respecto a los que nunca lo han hecho son residir en zona urbana, sexo (varón), tener padres divorciados, problemas con la educación, desempleo, problemas de integración social y problemas de salud. Encontramos, sin embargo, que entre aquellos que sólo han experimentado con el cannabis, los problemas psicológicos no discriminan entre los que lo usan y los que no" (Hammer y Vauglum, 1991, pp. 905-906). Otros factores encontrados en diferentes estudios son el inicio temprano al consumo, tener compañeros con conductas desviadas, tener padres consumidores de sustancias, salir frecuentemente de diversión, etc.

El gran objetivo de la prevención es retrasar la iniciación del uso de sustancias que son la "puerta de entrada" a otras drogas (Kaminer y Tarter, 1999). Si se previene el consumo de tabaco y alcohol incrementamos la probabilidad de un menor consumo de cannabis y, al tiempo, la prevención del consumo de cannabis previene el consumo de heroína o cocaína (Pentz, 1999). Sin embargo, también hay que tener en cuenta otras variables además del propio consumo o la "sustancia" en sí. Por ello, se puede concluir que: 1) existe una relación significativa entre el consumo 
de drogas legales (tabaco y alcohol) y el posterior consumo de cannabis, y entre el consumo de cannabis y el consumo posterior de heroína o cocaína; 2) aunque exista una relación no se deben confundir los términos de asociación y causalidad; 3) existen otras variables relacionadas con el consumo de cannabis y con el consumo de la heroína o cocaína, que en muchos casos pueden estar relacionadas con el consumo previo de estas sustancias, y que también pueden estar asociadas con el fenómeno de la escalada; 4) aun así, desde una perspectiva preventiva y de salud pública, hay que intervenir tanto sobre el cannabis, como sobre las otras variables que se relacionan con el consumo, tanto las sustancias previas en la cadena del consumo (ej., alcohol, tabaco), como en variables de tipo social (aceptación, disponibilidad), biológicas (predisposición) y psicológicas (ej., rasgos de personalidad, aprendizaje); 5) la prevención debe, por tanto, centrarse tanto en poner en marcha acciones para frenar el consumo de esas sustancias como para modificar aquellas variables que se relacionen con el inicio, la progresión y el mantenimiento del consumo de las distintas sustancias centrándose en las variables del individuo (ej., dotarlo de estrategias de afrontamiento) y del sistema social (ej., que tenga oportunidades educativas, laborales, etc.), así como en otras conductas relacionadas con el consumo de drogas (predisposición, conductas delictivas, baja autoestima, etc.).

Finalmente, hay que reconocer que se aprecia una importante laguna en la realización de estudios que tienen por objetivo analizar los factores de riesgo sobre el consumo de cannabis, a pesar de la clara importancia que ello tiene para la posterior predicción del consumo de otras drogas como la cocaína, las drogas de síntesis o la heroína. Quizás la causa de ello es la necesidad de tener que hacer estudios longitudinales de larga duración. No obstante, los resultados de las pocas investigaciones existentes, que con frecuencia han utilizado distintos tipos de diseños, son todos coincidentes entre sí, lo cual da robustez a los hallazgos de dichos estudios. Esto facilita poder diseñar programas preventivos para esta sustancia con una adecuada base empírica; esto es, basados en aquellos factores de riesgo y de protección que se han ido encontrando en los estudios existentes en la literatura científica.

\section{REFERENCIAS BIBLIOGRÁFICAS}

Bachman, J.G., Wadsworth, K.N., O'Malley, P.M. et al. (1997). Smoking, drinking, and drug use in young adulthood: The impacts of new freedoms and new responsibilities. Mahwah, $\mathrm{NJ}$ : Lawrence Earlbaum Associates.

Becoña, E. (1999). Bases teóricas que sustentan los programas de prevención de drogas. Madrid: Plan Nacional sobre Drogas.

Cabrera, R. y Torrecilla, J.M. (Coords.) (1998). Manual de drogodependencias. Madrid: Cauce Editorial.

Calafat, A., Bohrn, K., Juan, M. et al. (1999). Night life in Europe and recreative drugs use. SONAR 98. Palma de Mallorca: Irefrea, España.

Chen, K. y Kandel, D.B. (1998). Predictors of cessation of marihuana use: An event history analysis. Drug and Alcohol Dependence, 50, 109121.

Díaz, J. (1997). How drugs influence behavior. New Jersey: Prentice Hall.

Gold, M.S. y Tullis, M. (1999). Cannabis. En Galanter, M. y Kleber, H.D. (Eds.), Textbook of substance abuse treatment (2nd ed., pp. 165-181). Washington, DC: American Psychiatric Press.

Golub, A. y Johnson, B.D. (1994). The shifting importance of alcohol and marijuana as gateway substances among serious drug abusers. Journal of Studies on Alcohol, 55, 607-614.

Hammer, T. y Vaglum, P. (1991). Users and nonusers within a high risk milieu of cannabis use. A general population study. International Journal of Addictions, 26, 595-604.

Hulley, S. y Cummings, S.R. (1988). Designing clinical research. Baltimore: Williams \& Wilkins.

Kaminer, Y. y Tarter, R.E. (1999). Adolescent substance abuse. En M. Galanter y H.D. Kleber (Eds.), Textbook of substance abuse treatment (2nd. ed., pp. 465-474). Washington, DC: American Psychiatric Press. 
Kandel, D. (1975). Stages in adolescent involvement in drug use. Science, 190, 912-914.

Kandel, D.B. y Davies, M. (1992). Progresión to regular marijuana involvement: Phenomenology and risk factors for near-daily use. En M. Glantz y R. Pickens (Eds.), Vulnerability to drug abuse (pp. 211-253). Washington, D.C.: American Psychological Association.

Kandel, D.B. y Yamaguchi, K. (1985). Developmental patterns of the use of legal, illegal, and prescribed drugs. En C.L. Jones y R.J. Battjes (Eds.), Etiology of drug abuse (pp. 193-235). RockviIle, MD: National Institute on Drug Abuse.

Kandel, D. y Yamaguchi, K. (1999). Developmental stages of involvement in substance use. En P.J. Ott, R.E. Tarter y R.T. Ammerman (Eds.), Sourcebook on substance abuse. Etiology, epidemiology, assessment, and treatment (pp. 50-74). Nueva York: Allyn \& Bacon.

Kandel, D.B., Yamaguchi, K. y Chen, K. (1992). Stages of progresión in drug involvement from adolescent to adulthood: Further evidence for the gateway theory. Journal of Studies on Alcohol, 53, 447-457.

Kelly, TH., Foltin, R.W., Emurian, C.S. y Fischman, M.W. (1994). Effects of delta-9-THC on marijuana smoking, drug choice and verbal reports of drug liking. Journal of Experimental Analysis of Behavior, 61, 203-211.

Martin, B.R. (1999). Neurobiology of marihuana. En M. Galanter y H.D. Kleber (Eds.), Textbook of substance abuse treatment (2nd. ed., pp. 3946). Washington, DC: American Psychiatric Press.

Millman, R.B. y Beeder, A.B. (1997). Cannabis. En M. Galanter y H.D. Kleber (Eds.), El tratamiento de los trastornos por abuso de sustancias (pp. 91-109). Barcelona: Masson.

Moore, D.S. y McCabe, G.P. (1997). Introduction to the practice of statistics (2nd. ed.). Nueva York: W.H. Freeman and Company.

Neumark-Sztainer, D., Story, M., Dixon, L.B. y Murray, D.M. (1998). Adolescents engaging in unhealthy weight control behaviors: Are they at risk for other health-compromising behaviors? American Joumal of Public Health, 88, 952-955.

O'Donnell, J.A. y Clayton, R. (1982). The steppingstone hypothesis- Marijuana, heroin and causality. Chemical Dependence, 4, 229-241.
O'Malley, P.M., Johnston, L.D. y Bachman, J.G. (1999). Epidemiology of substance abuse in adolescence. En P.J. Ott, R.E. Tarter y R.T. Ammerman (Eds.), Sourcebook on substance abuse. Etiology, epidemiology, assessment, and treatment (2nd. ed., pp. 15-31). Boston: Allyn and Bacon.

Pentz, M.A. (1999). Prevention. En M. Galanter y H.D. Kleber (Eds.), Textbook of substance abuse treatment (2nd. ed., pp. 535-544). Washington, DC: American Psychiatric Press.

Plan Nacional sobre Drogas (1997). Encuesta sobre drogas a la población escolar. Madrid: Delegación del Gobierno para el Plan Nacional sobre Drogas.

Recio, J.L. (1995). The influence of family, school and peers on adolescent drug misuse. International Journal of Addictions, 30, 1407-1423.

Stenbacka, M., Allebeck, P., Brandt, L. y Romelsjö, A. (1992). Intravenous drug abuse in young men: Risk factors assessed in a longitudinal perspective. Scandinavian Journal of Social Medicine, 20, 94-101.

Stenbacka, M., Allenbeck, P. y Romelsjö, A. (1993). Initiation to drug abuse: The pathway from being offered drugs to trying cannabis and progression to intravenous drug abuse. Scandinavian Journal of Social Medicine, 21, 31-39.

Werch, C.E. y Anzalone, D. (1995). Stage theory and research on tobacco, alcohol, and other drug use. Journal of Drug Education, 25, 81-98.

Wills, T. A., McNamara, G., Vaccaro, D. y Hirky, E. (1997). Escalated substance use: A longitudinal grouping analysis from early to middle adolescence. En G.A. Marlatt y G.R. VandenBos (Eds.), Addictive behaviors: Readings on etiology, prevention, and treatment (pp. 97128). Washington, DC: American Psychological Association.

Yamaguchi, K. y Kandel, D. (1985). On the resolution of role incompatibility. A life event history of analysis of family roles and marihuana use. American Journal of Sociology, 90, 12841325.

Yu, J. y Williford, W. (1992). The analysis of drug use progression of young adults in New York State. The International Journal of the Addictions, 27, 1313-1323. 